OPEN ACCESS

Edited by:

Yun-Qing Li,

Fourth Military Medical University,

China

Reviewed by:

Jens Randel Nyengaard,

Aarhus University, Denmark

Ivan A. Lopez,

University of California, Los Angeles,

United States

*Correspondence:

Yong Tang

ytang062@163.com

tThese authors have contributed equally to this work.

Received: 16 September 2017 Accepted: 12 October 2017 Published: 17 November 2017

Citation:

Chen L, Zhou C, Tan C, Wang F,

Gao Y, Huang C, Zhang Y, Jiang L and Tang $Y$ (2017) Stereological

Study on the Positive Effect of Running Exercise on the Capillaries in

the Hippocampus in a Depression

Model.

Front. Neuroanat. 11:93. doi: 10.3389/fnana.2017.00093

\section{Stereological Study on the Positive Effect of Running Exercise on the Capillaries in the Hippocampus in a Depression Model}

\author{
Linmu Chen ${ }^{1 \dagger}$, Chunni Zhou ${ }^{1 \dagger}$, Chuanxue Tan ${ }^{1}$, Feifei Wang ${ }^{1}$, Yuan Gao ${ }^{2}$, \\ Chunxia Huang ${ }^{3}$, Yi Zhang ${ }^{1}$, Lin Jiang ${ }^{1}$ and Yong Tang ${ }^{1 *}$
}

${ }^{1}$ Department of Histology and Embryology, Chongqing Medical University, Chongqing, China, ${ }^{2}$ Department of Geriatrics, The First Affiliated Hospital, Chongqing Medical University, Chongqing, China, ${ }^{3}$ Department of Physiology, Chongqing Medical University, Chongqing, China

Running exercise is an effective method to improve depressive symptoms when combined with drugs. However, the underlying mechanisms are not fully clear. Cerebral blood flow perfusion in depressed patients is significantly lower in the hippocampus. Physical activity can achieve cerebrovascular benefits. The purpose of this study was to evaluate the impacts of running exercise on capillaries in the hippocampal CA1 and dentate gyrus (DG) regions. The chronic unpredictable stress (CUS) depression model was used in this study. CUS rats were given 4 weeks of running exercise from the fifth week to the eighth week (20 min every day from Monday to Friday each week). The sucrose consumption test was used to measure anhedonia. Furthermore, stereological methods were used to investigate the capillary changes among the control group, CUS/Standard group and CUS/Running group. Sucrose consumption significantly increased in the CUS/Running group. Running exercise has positive effects on the capillaries parameters in the hippocampal CA1 and DG regions, such as the total volume, total length and total surface area. These results demonstrated that capillaries are protected by running exercise in the hippocampal CA1 and DG might be one of the structural bases for the exercise-induced treatment of depression-like behavior. These results suggest that drugs and behavior influence capillaries and may be considered as a new means for depression treatment in the future.

Keywords: capillaries, depression-like behavior, hippocampus, exercise, stereology

\section{INTRODUCTION}

Depressive disorder is widely distributed in the population (Kessler et al., 2003), as it is the main reason of disability worldwide (Menard et al., 2016). Thus far, the depression theories of inflammatory (Maes et al., 2012), monoamine (Blier, 2003) and neurotrophic (Duman and Monteggia, 2006) compounds have been introduced by major researchers. Common antidepressant drugs act on monoamine neurotransmitters because of their significant role in the pathogenesis of depression. However, Malki et al. (2012) found that lag time always exists between the improvement of depressive symptoms and the restoration of the neurochemical balance, which means that the drug response of the primary targets might have comprehensive downstream biological events and that further studies are needed. 
To provide a new treatment for depression, it is important to further study the pathogenesis of depression.

Positive benefits in cognitive, emotional and motor domains combined with reductions in negative affect and distress can be acquired with regular physical exercise (Archer et al., 2014). Greist et al. (1979) revealed that aerobic running is a treatment for moderate depression. Some studies revealed that physical exercise might be a safe and effective antidepressant therapy in late-life major depression patients (Belvederi Murri et al., 2015) and in rats and mice with depression-like behavior (Lee et al., 2013; Eldomiaty et al., 2017). However, the mechanisms underlying the antidepressant effect of running exercise are not fully understood.

Blood vessels can be influenced by running exercise (Bolduc et al., 2013). Cerebrovascular benefits can be gained with regular engagement in physical activity (Guiney et al., 2015). The positive effects of running exercise on cerebral capillaries have been investigated by many studies. Kleim et al. (2002) found that independent running training for 30 days increased the capillary density of motor cortex in the 5-month-old male rats. Swain et al. (2003) found that after 30 days of running exercise, in 6- to 12-month-old rats the volume fraction of the cortex capillaries is increased in the II/III layer of the motor cortex. Using unbiased stereological methods, our previous work revealed that after running exercise the total length, total volume and total surface area of the capillaries in the cortex of aged rats (Huang et al., 2013) and in white matter in a rat model of depression (Chen et al., 2016) are increased. In treating vascular depression, greater depression reduction and lower rates of recurrence are found when combined antidepressant therapy with vasodilator drug (nimodipine), which supported the argument that cerebrovascular disease is related to the pathogenesis and recurrence of depression in patients (Taragano et al., 2001). As mentioned above, the change of capillaries might be involved in the pathogenesis of depression, and running exercise may reverse the symptoms of depression.

The knowledge gained from imaging research and the postmortem studies is catalyzing a paradigm shift in which primary mood disorders are conceptualized as illnesses that involve abnormalities in brain structure and function (Drevets, 2001). The hippocampus has a significant role in the feedback inhibition of the stress response (Yang et al., 2003). Autopsy results of depression patients confirm changes in the hippocampus (Duman et al., 2000). Using MRI, researchers found no significant difference in the whole brain volume between depression patients and normal individuals, but the hippocampal volume was significantly smaller in depression patients than in normal individuals (Nifosi et al., 2010; Ahdidan et al., 2011; Amico et al., 2011). Therefore, the hippocampus plays an important role in the process of depression, but the specific mechanism remains to be further studied.

Nevertheless, whether vascular change in hippocampus is involved in the treatment effect of running exercise on depression-like behavior in rat is still unknown. We hypothesized that the microvascular system in the hippocampus in depression model undergoes significantly change before and after running exercise. To test this hypothesis, we established an effective and feasible depression animal model via chronic unpredictable stress (CUS) stimuli (Manosso et al., 2016). After running exercise, a stereological quantitative method was used to investigate the changes in capillaries in hippocampal CA1 and dentate gyrus (DG) regions in a rat model of depression.

\section{MATERIALS AND METHODS}

\section{Animals}

The Experimental Animal Center of Chongqing Medical University provided the male Sprague-Dawley rats. The rats were acclimated to their surroundings for 1 week before experimentation. They were individually housed at a temperature of $22 \pm 2{ }^{\circ} \mathrm{C}$, a humidity of $55 \pm 10 \%$ and kept under a constant $12 \mathrm{~h}$ light-12 $\mathrm{h}$ dark cycle. According to the open field test, 100 rats were picked up for the next experiment. This study was carried out in accordance with the recommendations of Chongqing Management Approach of Laboratory Animal (Chongqing government order No.195). The protocol was approved by the Institutional Review Board of Chongqing Medical University (Reference Number: CQMU 2010-26).

\section{Establishment of the Depression Model and Running Exercise Protocol}

The main CUS process was described in a previous study (Chen et al., 2016). The basic steps are as follows: the rats were randomly divided into two groups: control group $(n=10)$ and CUS group $(n=90)$. Each rat in the CUS group was given CUS stimuli while housed in a single cage. The rats in the control group were housed 5 rats per cage and were not given CUS stimuli. Each random stimulus did not appear continuously. Then, 20 rats with significantly lower sucrose consumption were selected from the CUS group and divided into a CUS/Standard group $(n=10)$ and a CUS/Running group $(n=10)$. The rats in the CUS/Running group were forced to run on a six-lane motorized treadmill. The exercise protocol was similar to that of a previous study (Chen et al., 2016).

\section{Behavior Test}

Anhedonia, the core symptom of depression in humans (Scheggi et al., 2015), can be measured via a sucrose consumption test (Hurley et al., 2014). The detailed protocol was described in a previous report (Hurley et al., 2014). The sucrose consumption test was carried out every Saturday at 7 am for 8 weeks.

The general exploratory behavior and locomotor behavior were evaluated by open field test. The test was operated as described previously with modifications (Duman et al., 2008).

\section{Tissue Processing}

First, the rats were anesthetized with $1 \%$ pentobarbital sodium (4 ml/kg). Then, 4\% paraformaldehyde was diluted with PBS $(\mathrm{pH}=7.4)$, and $0.9 \%$ heparinized saline was perfused intracardially in left ventricular apex in sequence. After that, 


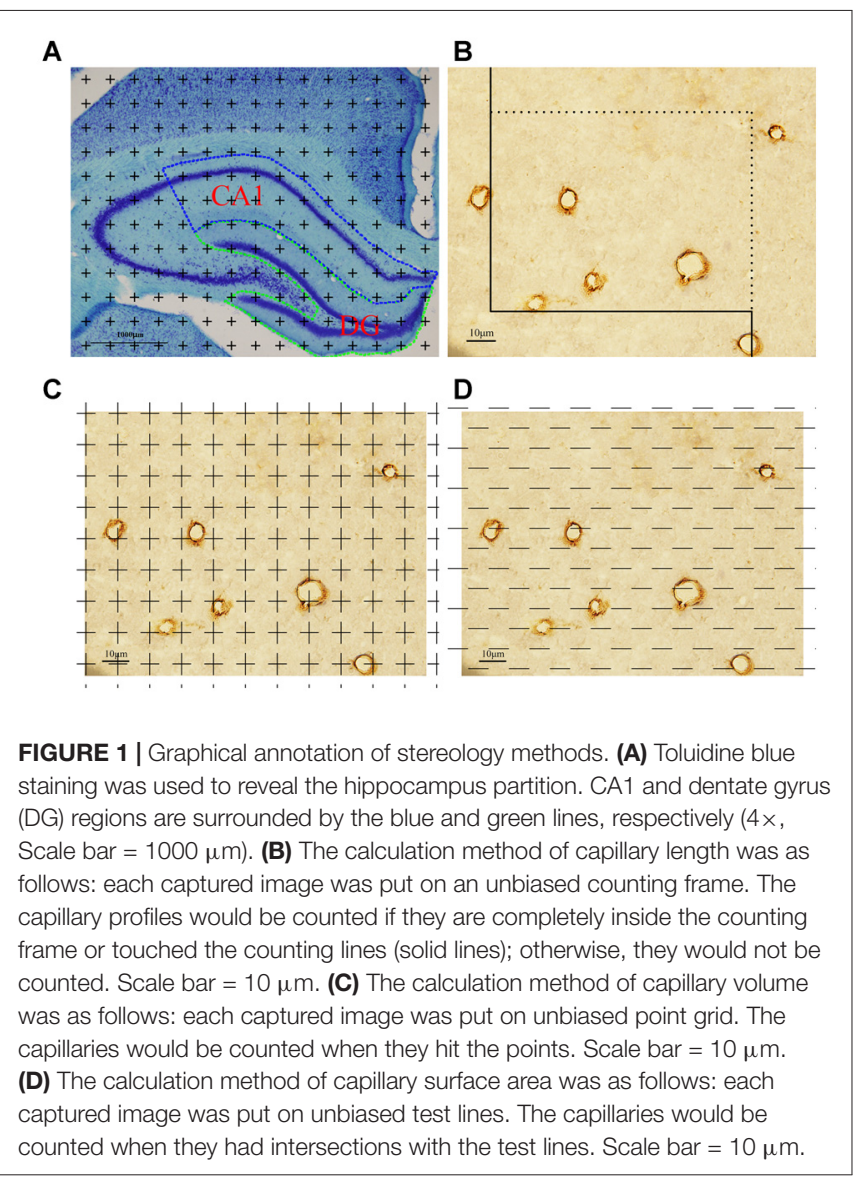

the cerebrum was obtained and cut into two hemispheres. Each hemisphere was sliced into $1 \mathrm{~mm}$ consecutive slabs coronally.

\section{Estimation of the CA1 and DG Region Volumes}

One $10-\mu \mathrm{m}$-thick frozen slice was obtained from each $1 \mathrm{~mm}$ slab. Toluidine blue staining was done to mark the area of CA1 and DG region (Figure 1A). The total CA1 and DG region volumes in each $1 \mathrm{~mm}$ slab were calculated through Cavalieri's principle (Chao et al., 2015). A brief description is as follows: the caudal surface of each slab was hit by randomly equidistant points in the transparent plastic sheet and the points hitting the object region were counted. The calculation formula was as follows (Yang et al., 2015):

$$
\mathrm{V}_{(\mathrm{CA} 1)}=\mathrm{t} \times \mathrm{a}(\mathrm{p}) \times \sum \mathrm{P}_{(\mathrm{CA} 1)} \times 2
$$

t means the slab thickness $(1 \mathrm{~mm}), \mathrm{a}(\mathrm{p})$ means the area associated with each point and $\sum \mathrm{P}_{(\mathrm{CA} 1)}$ means the total number of points hitting the CA1 per hemisphere.

The DG region volume was similarly calculated using the above methods.

\section{Sampling from the CA1 and DG Regions and Immunohistochemical Staining}

Sampling methods were described by our previous work (Chen et al., 2016). The obtained slabs were embedded in optimal cutting temperature compound (OCT) to obtain frozen sections, and the sector technique was used to produce isotropic and uniformly random (IUR) sections (Chao et al., 2015). The thickness of the frozen section is $4 \mu \mathrm{m}$. On average, 20 frozen sections could be obtained per rat.

A brief description of immunohistochemical staining is as follows: each slice was fixed in acetone for $10 \mathrm{~min}$ and put in a high temperature in citrate solution for $15 \mathrm{~min}$ to repair antigens. Then, $3 \%$ hydrogen peroxide for $15 \mathrm{~min}$ at $37^{\circ} \mathrm{C}$ was used to inactivate endogenous peroxides, and nonspecific

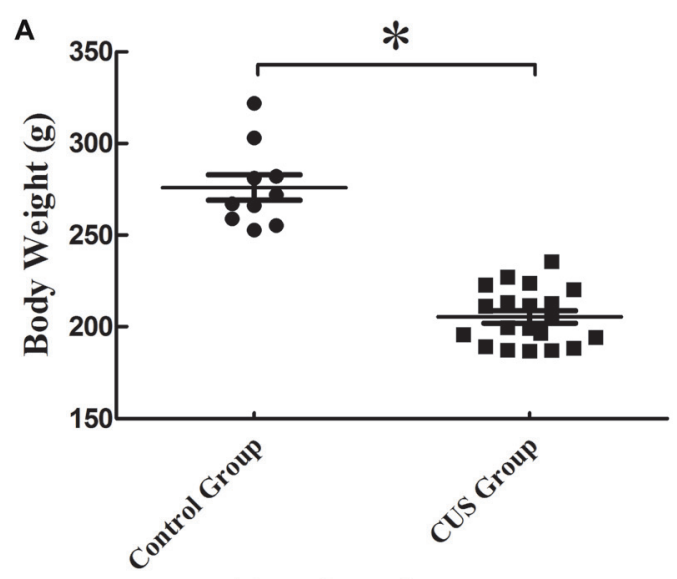

Fourth week

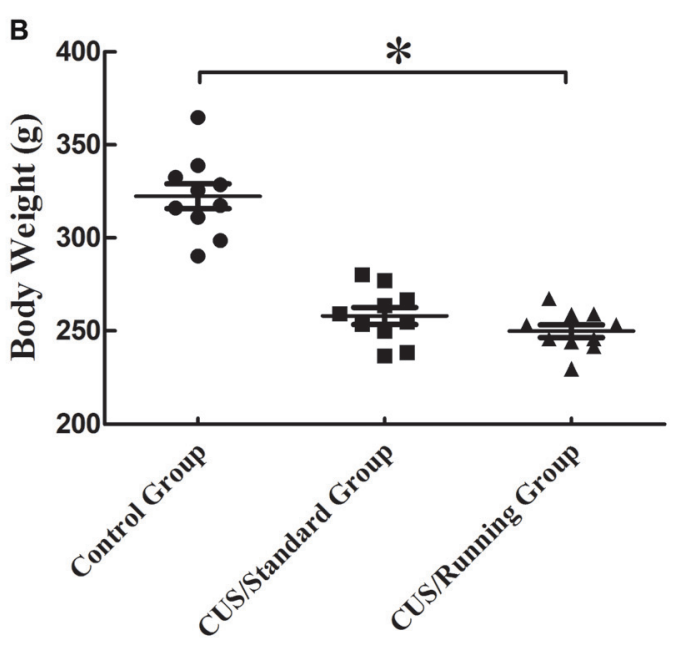

Eighth week

FIGURE 2 | Changes of body weight before and after running exercise. (A) The comparison of body weight between the CUS group and control group in the procedure of CUS stimuli in the fourth week. (B) Effect of running exercise on the body weight among the three groups. *Indicates $p<0.05$, compared with the control group. 


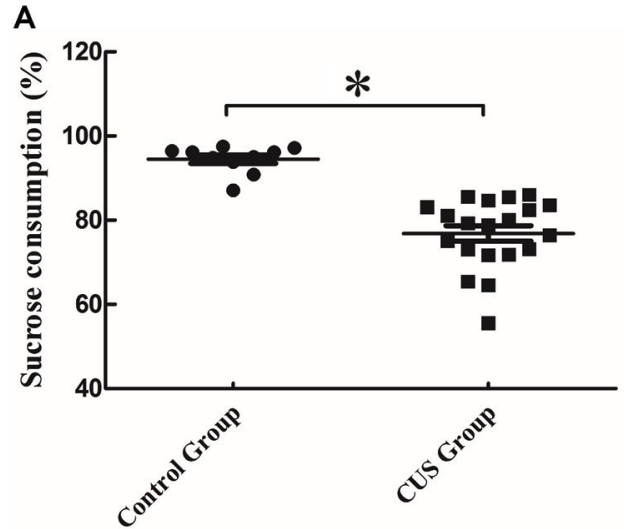

Fourth week

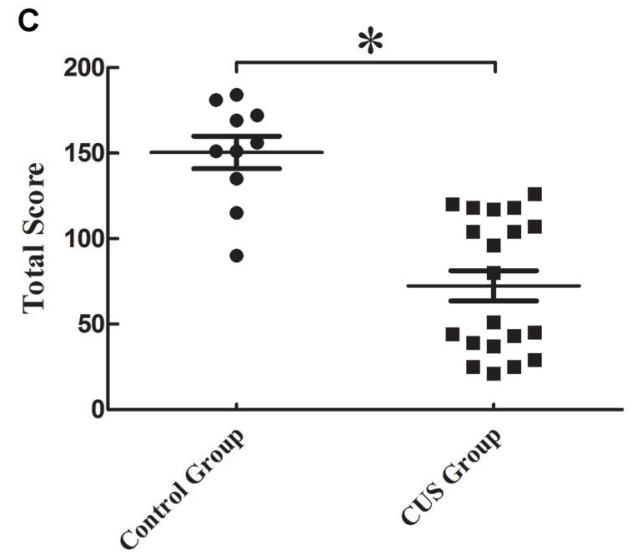

Fourth week

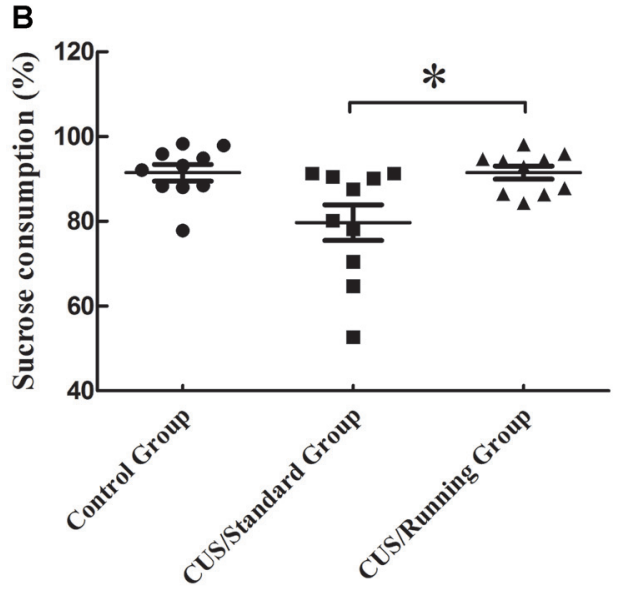

Eighth week

D

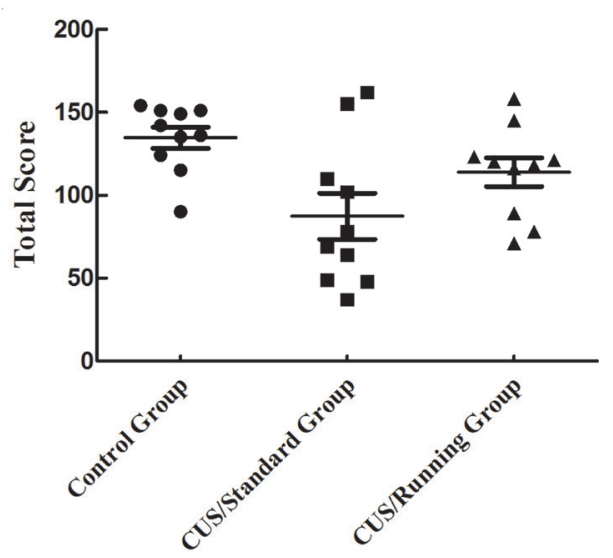

Eighth week

FIGURE 3 | Changes of behavior tests before and after running exercise. (A,C) The results of sucrose consumption and open field test in the CUS group and control group after the procedure of CUS stimuli. (B,D) The results of sucrose consumption and open field test among the control group, CUS/Standard group and the CUS/Running group after running exercise. ${ }^{*}$ Indicates $p<0.05$.

staining was blocked by normal goat serum for $30 \mathrm{~min}$ at $37^{\circ} \mathrm{C}$. Mouse monoclonal CD31+ (Abcam, UK; Albelda et al., 1991) was incubated at $4^{\circ} \mathrm{C}$ overnight. Subsequently, the slice was transferred to a specific secondary antibody solution for $30 \mathrm{~min}$ at $37^{\circ} \mathrm{C}$ and diaminobenzidine (DAB) solution was used to display color. After the above procedure, the section was observed using a light microscope of a stereological system. The vessels with a luminal diameter of less than $10 \mu \mathrm{m}$ were defined as components of the capillary network (Alba et al., 2004).

\section{Total Length of the Capillaries in CA1}

Every captured photograph was randomly superimposed by an unbiased counting frame (Figure 1B). The length density of the capillaries in the CA1 was analyzed with a method previously described (Shao et al., 2010):

$$
\mathrm{L}_{\mathrm{V}}\left(\mathrm{cap} / \mathrm{CA}_{1}\right)=2 \times \sum \mathrm{Q}\left(\mathrm{cap} / \mathrm{CA}_{1}\right) / \sum \mathrm{A}
$$

$\Sigma \mathrm{Q}$ (cap/CA1) means the total number of the capillary profiles in CA1 counted per rat. $\Sigma A$ means the total area of the counting frames used per rat. The total length of the capillaries in the CA1 could be obtained by multiplying the length density of the capillaries in the CA1, $\mathrm{L}_{\mathrm{V}}(\mathrm{cap} / \mathrm{CA1})$, by the CA1 volume $\left(\mathrm{V}_{(\mathrm{CA1})}\right)$.

\section{Total Volume of the Capillaries in CA1}

Every captured photograph was randomly placed by a transparent point grid (Figure 1C). The volume fraction of the capillaries in the $\mathrm{CA} 1, \mathrm{~V}_{\mathrm{V}}(\mathrm{cap} / \mathrm{CA1})$, was analyzed using the Following formula (Shao et al., 2010):

$$
\mathrm{V}_{\mathrm{V}}\left(\mathrm{cap} / \mathrm{CA}_{1}\right)=\sum \mathrm{P}\left(\mathrm{cap} / \mathrm{CA}_{1}\right) / \sum \mathrm{P}\left({ }_{\mathrm{CA} 1}\right)
$$

The total volume of the capillaries in the CA1 was analyzed by multiplying the volume fraction of the capillaries in the $C A 1, V_{V}$ (cap/CA1), by the CA1 volume $\left(\mathrm{V}_{(\mathrm{CA} 1)}\right)$. 

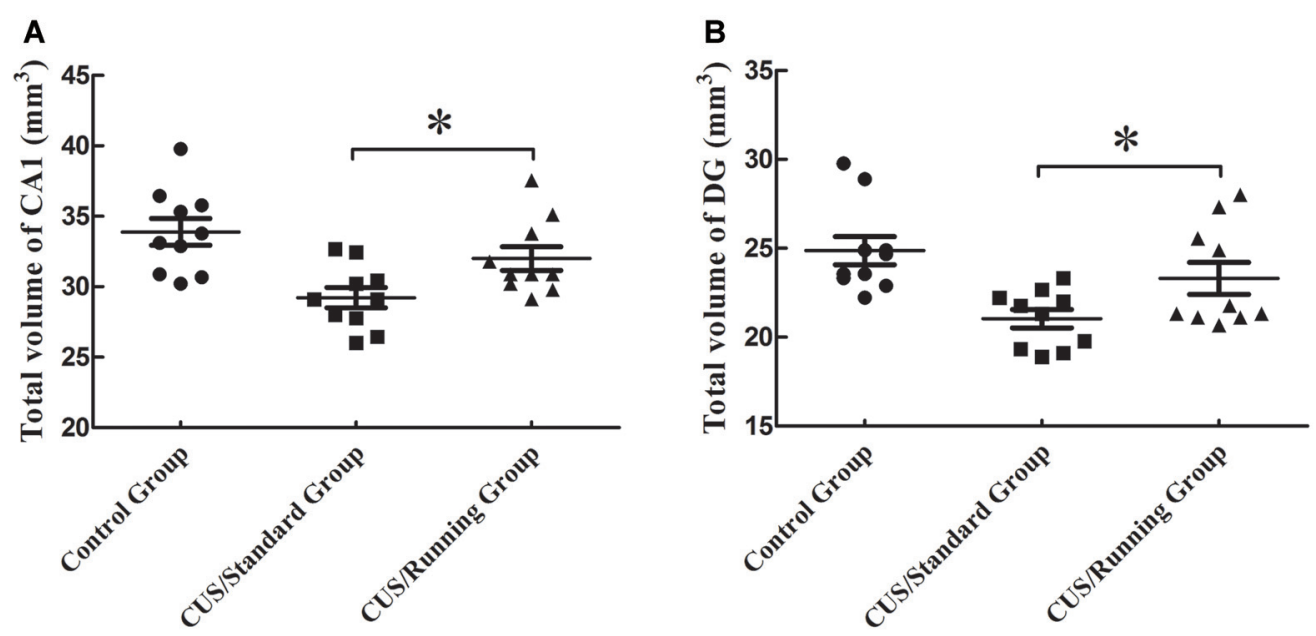

FIGURE 4 | Influence of running exercise on the total volume in the CA1 and DG regions. (A) Comparison of the volume (mean \pm SD) in CA1 among the control group, CUS/Standard group and the CUS/Running group. (B) Comparison of the volume (mean \pm SD) in the DG region among the control group, CUS/Standard group and the CUS/Running group. *Indicates $p<0.05$.

\section{Total Surface Area of the Capillaries in CA1}

Every captured photograph was randomly placed by grids of test lines (Figure 1D). The surface area density of the capillaries in the CA1 was calculated using the following formula (Shao et al., 2010):

$$
\mathrm{S}_{\mathrm{V}}(\operatorname{cap} / \mathrm{CA1})=2 \times \sum \mathrm{I}\left(\operatorname{cap}_{(\mathrm{CA} 1)}\right) / \sum \mathrm{L}
$$

The total surface area of the capillaries in the CA1 was analyzed by multiplying SV (cap/ $\left.{ }_{(\mathrm{CA} 1)}\right)$ by the CA1 volume $\left(\mathrm{V}_{(\mathrm{CA})}\right)$.

The total length, total volume and total surface area of the capillaries in the DG region were estimated using the same methods described above.

\section{Statistics}

Data are expressed as the mean \pm standard deviation (SD). Statistical analysis was performed for each of the dependent variables on the groups using analysis of variance (ANOVA). Group differences for these parameters were assessed using statistical product and service solutions software, with $p<0.05$ as the criterion for statistical significance.

\section{RESULTS}

\section{Running Exercise Does Not Alter the Rats' Body Weight in the CUS/Running Group}

After 4 weeks of CUS stimuli, the body weight significantly decreased in the CUS group compared with that in the control group ( $p<0.05$; Figure 2A). However, 4 weeks of running exercise did not increase the body weight in the CUS/Running group. The body in the CUS/Running group was still lower than that in the control group $(p<0.05$; Figure 2B).

\section{Running Exercise Increases the Sucrose Consumption in the CUS/Running Group}

After 4 weeks of CUS stimuli, the percentage of sucrose consumption in the CUS group ((76.9 \pm 8.16) \%) was significantly lower than that in the control group $((94.5 \pm 3.24) \% ; p<0.05 ;$ Figure 3A). This finding indicates that the construction of the depression model was successful. After the 4-week running period, the percentage of sucrose consumption in the CUS/Running group was elevated ((91.5 \pm 4.83$) \%)$ and much higher than that in the CUS/Standard group $((79.7 \pm 13.3) \%)$ $(p<0.05 ;$ Figure 3B). The sucrose consumption test reflects the anhedonia. Therefore, our results demonstrated that 4 weeks of running exercise reversed depressive behaviors in rats. However, the results of another behavior test, the open field test, showed that CUS stimuli could significantly reduce the total score in the CUS group $(p<0.05$; Figure 3C), but running exercise could not largely increase the total score in the CUS/Running group (Figure 3D).

\section{Running Exercise Significantly Increases the Volume of CA1 and DG Regions}

The accurate results of CA1 total volume were $33.9 \pm 3.01 \mathrm{~mm}^{3}$ in the control group, $29.2 \pm 2.27 \mathrm{~mm}^{3}$ in the CUS/Standard group and $32.0 \pm 2.66 \mathrm{~mm}^{3}$ in the CUS/Running group. Statistical analysis showed that running exercise significantly increased the CA1 total volume in the CUS/Running group compared with that in the CUS/Standard group (Figure 4A).

Similarly, the accurate results of DG region total volume were $24.9 \pm 2.52 \mathrm{~mm}^{3}$ in the control group, $21.0 \pm 1.62 \mathrm{~mm}^{3}$ in the CUS/Standard group and $23.3 \pm 2.84 \mathrm{~mm}^{3}$ in the CUS/Running group. The total volume of the DG 

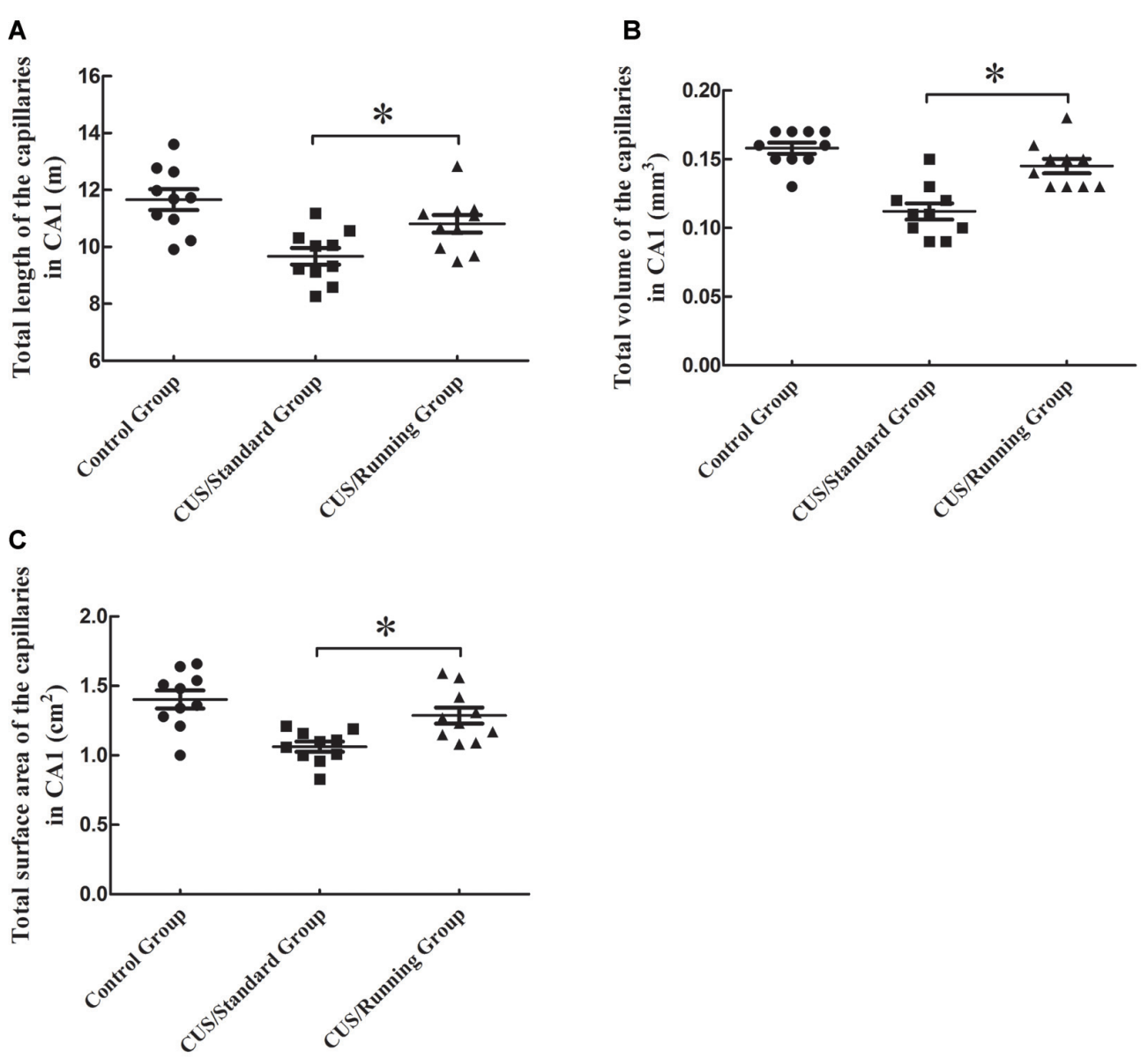

FIGURE $\mathbf{5}$ | Influence of running exercise on the capillary parameters in the CA1. (A) Comparison of the capillaries' total lengths in CA1 among the control group, CUS/Standard group and the CUS/Running group. (B) Comparison of the capillaries' total volume in CA1 among the control group, CUS/Standard group and the CUS/Running group. (C) Comparison of the capillaries' total surface area in CA1 among the control group, CUS/Standard group and the CUS/Running group. *Indicates $P<0.05$.

region was significantly increased in the CUS/Running group compared with that in the CUS/Standard group (Figure 4B).

\section{Changes in the Total Length, Total Volume and Total Surface Area of the Capillaries in CA1}

The total lengths of the capillaries in the CA1 were $11.7 \pm 1.15 \mathrm{~m}$ in the control group, $9.67 \pm 0.92 \mathrm{~m}$ in the CUS/Standard group and $10.8 \pm 0.976 \mathrm{~m}$ in the CUS/Running group. Running exercise significantly increased the total length of the capillaries in the CA1 in the CUS/Running group compared with that in the CUS/Standard group (Figure 5A).

The total volumes of the capillaries in the CA1 were $0.157 \pm 0.0130 \mathrm{~mm}^{3}$ in the control group, $0.111 \pm 0.0180 \mathrm{~mm}^{3}$ in the CUS/Standard group and $0.144 \pm 0.0160 \mathrm{~mm}^{3}$ in the CUS/Running group. Running exercise significantly increased the total length of the capillaries in the CA1 in the
CUS/Running group compared with that in the CUS/Standard group (Figure 5B).

The total surface areas of the capillaries in the CA1 were $1.40 \pm 0.204 \mathrm{~cm}^{2}$ in the control group, $1.06 \pm 0.117 \mathrm{~cm}^{2}$ in the CUS/Standard group and $1.29 \pm 0.184 \mathrm{~cm}^{2}$ in the CUS/Running group. Running exercise significantly increased the total length of the capillaries in the CA1 in the CUS/Running group compared with that in the CUS/Standard group (Figure 5C).

However, both of the above three parameters in CA1 showed no differences between the control group and CUS/Running group $(p>0.05)$.

\section{Changes in the Total Length, Total Volume and Total Surface Area for the Capillaries in the DG Region}

The total lengths of the capillaries in the DG region were $10.9 \pm 1.03 \mathrm{~m}$ in the control group, $9.38 \pm 0.688 \mathrm{~m}$ in the CUS/Standard group and $10.4 \pm 0.979 \mathrm{~m}$ in the 

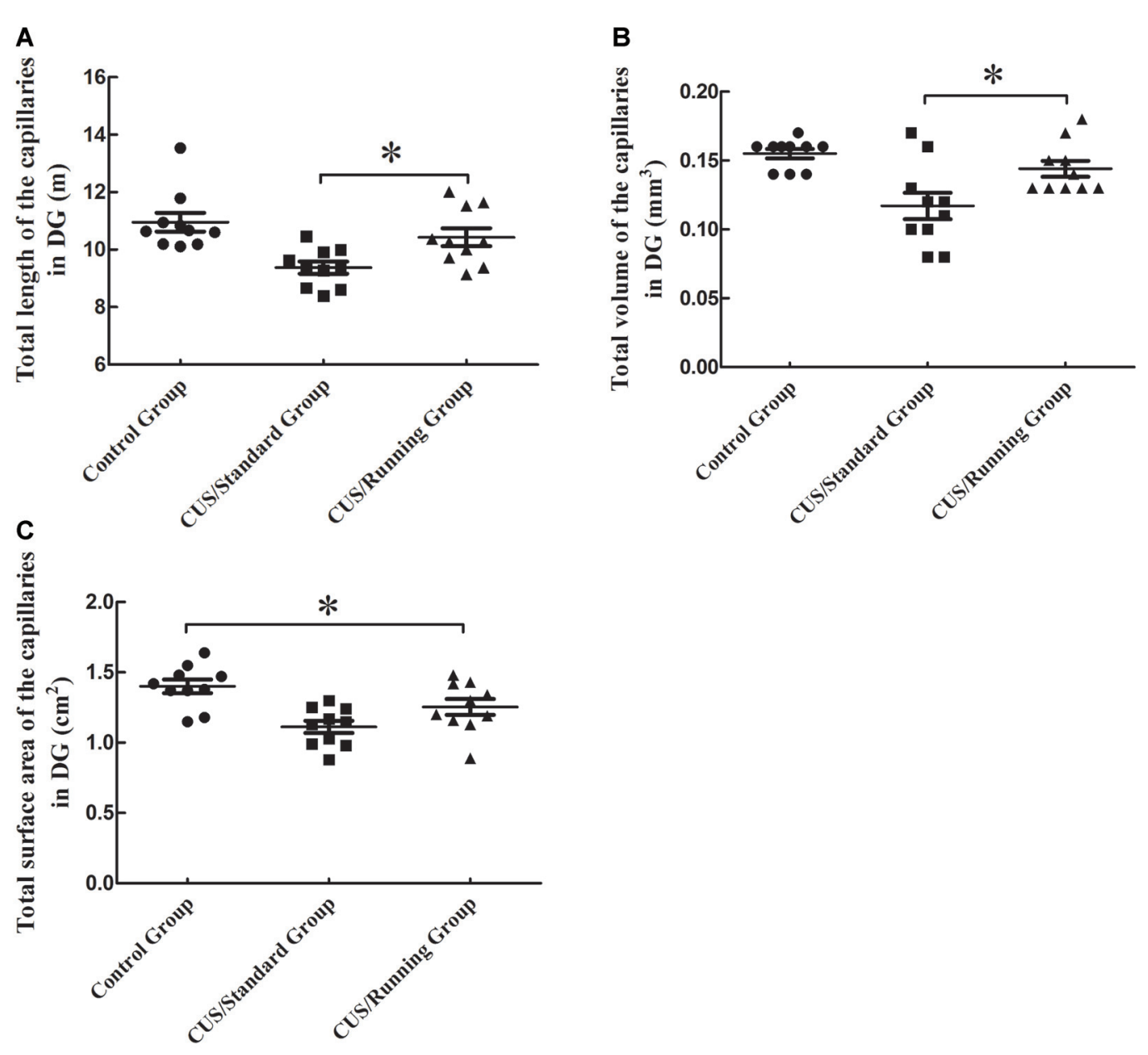

FIGURE 6 | Influence of running exercise on the capillary parameters in DG region. (A) Comparison of the capillaries' total lengths in DG region among the control group, CUS/Standard group and the CUS/Running group. (B) Comparison of the capillaries' total volume in the DG region among the control group, CUS/Standard group and the CUS/Running group. (C) Comparison of the capillaries' total surface area in the DG region among the control group, CUS/Standard group and the CUS/Running group. ${ }^{*}$ Indicates $P<0.05$.

CUS/Running group. Running exercise significantly increased the total length of the capillaries in the CA1 in the CUS/Running group compared with that in the CUS/Standard group (Figure 6A).

The total volumes of the capillaries in the DG region were $0.155 \pm 0.0110 \mathrm{~mm}^{3}$ in the control group, $0.115 \pm 0.0300 \mathrm{~mm}^{3}$ in the CUS/Standard group and $0.143 \pm 0.0180 \mathrm{~mm}^{3}$ in the CUS/Running group. Running exercise significantly increased the total length of the capillaries in the DG region in the CUS/Running group compared with that in the CUS/Standard group (Figure 6B).

The total surface areas of the capillaries in the DG region were $1.40 \pm 0.151 \mathrm{~cm}^{2}$ in the control group, $1.11 \pm 0.135 \mathrm{~cm}^{2}$ in the CUS/Standard group and $1.25 \pm 0.177 \mathrm{~cm}^{2}$ in the CUS/Running group. Statistical analysis showed no difference between the CUS/Running group and the CUS/Standard group, but the total surface area of the capillaries in the CUS/Running group was still lower than that in the control group (Figure 6C), which means running exercise had no significant effect on this parameter in the DG region.

\section{DISCUSSION}

Our study displayed that the preference of sucrose consumption in CUS/Running group was higher than that in the CUS/Standard group and reached normal levels when compared with the control group. Our results further confirmed that running had a therapeutic effect on the depression symptom. This research simulated the process of the development of human depression using the CUS depression model. Our study showed that the preference of sucrose consumption and the open-field score were decreased significantly in the CUS group after 4 weeks, which indicated that the depression model was established successfully. Running exercise, which is a safe, economic and simple means of behavior, has been successfully applied to the treatment of various diseases (Weuve et al., 
2004; Ravaglia et al., 2008). Greist et al. (1979) reported that running exercise has the same therapeutic effect on depression compared with the traditional psychological treatment for the first time. Recently, clinical studies and animal experiments also demonstrated that running exercise is conducive to the rehabilitation of depression (Zheng et al., 2006; Ensari et al., 2014). However, Chalder et al. (2012) found that the addition of a facilitated physical activity intervention to usual care did not improve depression outcome or reduce use of antidepressants compared with usual care alone. We speculated that the following reasons might partly explain the different outcomes. First, Beck depression inventory scores of 361 adults aged 18-69 in the study of Chalder et al. (2012) are 14 or more. These Beck depression inventory scores indicated that the depression symptoms of the participants in their study were moderate or severe with different types (Beck et al., 1996). However, it is generally believed that running exercise has a positive effect on mild or moderate depression alone (McCurdy et al., 2017). Second, the intensity and duration of running exercise can be easily controlled in animal study. However, in human study, as Chalder et al. (2012) claimed in the discussion part, the basis of participants' interest or motivation to engage in physical activity were not taken in to account. In human study, physical activity is notoriously difficult to measure and the self-reported assessment could have been biased by knowledge of the treatment allocation. To some degree, the participants' knowledge of their treatment allocation influenced their responses. Therefore, we thought that the actual amount and time of exercise in the study of depression patients might be different from those in the regular exercise studies on the animals with the depression-like behaviors, which might have important impact on the overall results.

The hippocampal formation is composed of hippocampal gyrus (CA1, CA2 and CA3), DG and subiculum. The CA1 and DG are two significant parts of the hippocampal formation (Yaghmaei et al., 2009). With a deeper understanding of the pathogenesis of depression, researchers found that the whole brain volume in depression patients is not significantly different from that in normal subjects, but the hippocampus volume is significantly lower (Nifosi et al., 2010; Ahdidan et al., 2011). After 4 weeks of running, the volumes of the CA1 area and DG region are significantly increased in the CUS/Running group compared with those in the CUS/Standard group. However, the exact cellular mechanism behind the volume changes remains unknown. Degenerative changes in capillary seem to be one of the initial factors of nervous system diseases (Riddle et al., 2003). The incomplete function and structure of capillary can affect the support of oxygen and blood, leading to lower metabolism and chronic hypoperfusion (Farkas and Luiten, 2001).

With the development of imaging technology, researchers make dynamic observation of the blood flow in living brain, the chemical activity and metabolism of neuron systems, which provide much more support for the study of the vascular mechanism of depression (Niell and Smith, 2004). In the process of the treatment of depression, the researchers found that the vascular disease has also been improved (Otte et al., 2007) and augmentation of antidepressant therapy with vasodilators leads to greater depression reduction and lower rates of recurrence (Farkas and Luiten, 2001). These studies indicated that depression and cardiovascular diseases may have a common mechanism. Based on the above research results, we presumed that improving blood vessels may be a way of treating depression. Black et al. (1990) found that running exercise led to an increase in vascular density in the paramedian lobule of adult rats. After running exercise, the vascular density increased because of the metabolic need (Isaacs et al., 1992). However, most of these studies only observed blood flow changes in the brain or in a region of the brain after exercise using an imaging technique or other techniques. A capillary is a three-dimensional structure, and the distribution in the brain is extremely irregular; thus, different results could be obtained either from different biopsy parts or different directions. Moreover, the density value, which is the two-dimensional plane information, cannot reflect the real total blood capillary. Modern stereological methods are used to obtain accurate three-dimensional structure morphometric features (Tang et al., 2009), which provide good tools for the precise quantitative date of the capillaries. Using the stereological methods, we obtained accurate quantitative results of capillaries parameters in the CA1 and DG regions after running exercise, which provided a basic parameter for the treatment of depression.

In summary, our experiment accurately measured the basic parameters of hippocampus capillaries in CUS rats after running exercise, such as the total volume, total length and total surface area of capillary. Our result was consistent with the theory of "vascular depression". It demonstrated that the depression rats had a change in the vascular structure and running exercise was an effective treatment for depression symptoms through reversing the decline of the parameters of the capillaries in the hippocampus. Therefore, drugs and behavior, which can influence capillaries, might be a new direction for the treatment of depression in the future.

\section{AUTHOR CONTRIBUTIONS}

All authors have full access to all research data and are responsible for the accuracy of data integrity and data analysis. LC, CT and YT: conception and design of the article. FW, YG and $\mathrm{CH}$ : performance of animal experiment and behavior tests. YZ, LJ and CZ: immumohistochemical staining. LC: analysis of the data. LC, CT and YT: writing and editing of the article.

\section{FUNDING}

This study was supported by the National Natural Science Foundation of China (NSFC, 31300985; NSFC, 81501156; NSFC, 81671259).

\section{ACKNOWLEDGMENTS}

We would like to thank professor Lei Zhang and Fenglei Chao for their assistance. 


\section{REFERENCES}

Ahdidan, J., Hviid, L. B., Chakravarty, M. M., Ravnkilde, B., Rosenberg, R., Rodell, A., et al. (2011). Longitudinal MR study of brain structure and hippocampus volume in major depressive disorder. Acta Psychiatr. Scand. 123, 211-219. doi: 10.1111/j.1600-0447.2010.01644.x

Alba, C., Vidal, L., Diaz, F., Villena, A., and de Vargas, I. P. (2004). Ultrastructural and quantitative age-related changes in capillaries of the dorsal lateral geniculate nucleus. Brain Res. Bull. 64, 145-153. doi: 10.1016/j.brainresbull. 2004.06.006

Albelda, S. M., Muller, W. A., Buck, C. A., and Newman, P. J. (1991). Molecular and cellular properties of PECAM-1 (endoCAM/CD31): a novel vascular cell-cell adhesion molecule. J. Cell Biol. 114, 1059-1068. doi: 10.1083/jcb.114. 5.1059

Amico, F., Meisenzahl, E., Koutsouleris, N., Reiser, M., Möller, H. J., and Frodl, T. (2011). Structural MRI correlates for vulnerability and resilience to major depressive disorder. J. Psychiatry Neurosci. 36, 15-22. doi: 10.1503/jpn.090186

Archer, T., Josefsson, T., and Lindwall, M. (2014). Effects of physical exercise on depressive symptoms and biomarkers in depression. CNS Neurol. Disord. Drug Targets 13, 1640-1653. doi: 10.2174/1871527313666141130203245

Beck, A. T., Steer, R. A., and Brown, G. K. (1996). Manual for the Beck Depression Inventory-II. San Antonio, TX: Psychological Corporation.

Belvederi Murri, M., Amore, M., Menchetti, M., Toni, G., Neviani, F., Cerri, M., et al. (2015). Physical exercise for late-life major depression. Br. J. Psychiatry 207, 235-242. doi: 10.1192/bjp.bp.114.150516

Black, J. E., Isaacs, K. R., Anderson, B. J., Alcantara, A. A., and Greenough, W. T. (1990). Learning causes synaptogenesis, whereas motor activity causes angiogenesis, in cerebellar cortex of adult rats. Proc. Natl. Acad. Sci. U S A 87, 5568-5572. doi: 10.1073/pnas.87.14.5568

Blier, P. (2003). The pharmacology of putative early-onset antidepressant strategies. Eur. Neuropsychopharmacol. 13, 57-66. doi: 10.1016/s0924977x $(02) 00173-6$

Bolduc, V., Thorin-Trescases, N., and Thorin, E. (2013). Endothelium-dependent control of cerebrovascular functions through age: exercise for healthy cerebrovascular aging. Am. J. Physiol. Heart Circ. Physiol. 305, H620-H633. doi: 10.1152/ajpheart.00624.2012

Chalder, M., Wiles, N. J., Campbell, J., Hollinghurst, S. P., Haase, A. M., Taylor, A. H., et al. (2012). Facilitated physical activity as a treatment for depressed adults: randomised controlled trial. BMJ 344:e2758. doi: 10.1136/bmj.e2758

Chao, F., Zhang, L., Luo, Y., Xiao, Q., Lv, F., He, Q., et al. (2015). Running exercise reduces myelinated fiber loss in the dentate gyrus of the hippocampus in APP/PS1 transgenic mice. Curr. Alzheimer Res. 12, 377-383. doi: $10.2174 / 1567205012666150325183011$

Chen, L.-M., Zhang, A.-P., Wang, F.-F., Tan, C.-X., Gao, Y., Huang, C.-X., et al. (2016). Running exercise protects the capillaries in white matter in a rat model of depression. J. Comp. Neurol. 524, 3577-3586. doi: 10.1002/cne. 24017

Drevets, W. C. (2001). Neuroimaging and neuropathological studies of depression: implications for the cognitive-emotional features of mood disorders. Curr. Opin. Neurobiol. 11, 240-249. doi: 10.1016/s0959-4388(00)00203-8

Duman, R. S., Malberg, J., Nakagawa, S., and D'Sa, C. (2000). Neuronal plasticity and survival in mood disorders. Biol. Psychiatry 48, 732-739. doi: 10.1016/s0006-3223(00)00935-5

Duman, R. S., and Monteggia, L. M. (2006). A neurotrophic model for stressrelated mood disorders. Biol. Psychiatry 59, 1116-1127. doi: 10.1016/j.biopsych. 2006.02.013

Duman, C. H., Schlesinger, L., Russell, D. S., and Duman, R. S. (2008). Voluntary exercise produces antidepressant and anxiolytic behavioral effects in mice. Brain Res. 1199, 148-158. doi: 10.1016/j.brainres.2007.12.047

Eldomiaty, M. A., Almasry, S. M., Desouky, M. K., and Algaidi, S. A. (2017). Voluntary running improves depressive behaviours and the structure of the hippocampus in rats: a possible impact of myokines. Brain Res. 1657, 29-42. doi: 10.1016/j.brainres.2016.12.001

Ensari, I., Motl, R. W., and Pilutti, L. A. (2014). Exercise training improves depressive symptoms in people with multiple sclerosis: results of a metaanalysis. J. Psychosom. Res. 76, 465-471. doi: 10.1016/j.jpsychores.2014. 03.014
Farkas, E., and Luiten, P. G. (2001). Cerebral microvascular pathology in aging and Alzheimer's disease. Prog. Neurobiol. 64, 575-611. doi: 10.1016/s03010082(00)00068-x

Greist, J. H., Klein, M. H., Eischens, R. R., Faris, J., Gurman, A. S., and Morgan, W. P. (1979). Running as treatment for depression. Compr. Psychiatry 20, 41-54. doi: 10.1016/0010-440x(79)90058-0

Guiney, H., Lucas, S. J., Cotter, J. D., and Machado, L. (2015). Evidence cerebral blood-flow regulation mediates exercise-cognition links in healthy young adults. Neuropsychology 29, 1-9. doi: 10.1037/neu0000124

Huang, C. X., Qiu, X., Wang, S., Wu, H., Xia, L., Li, C., et al. (2013) Exercise-induced changes of the capillaries in the cortex of middle-aged rats. Neuroscience 233, 139-145. doi: 10.1016/j.neuroscience.2012.12.046

Hurley, L. L., Akinfiresoye, L., Kalejaiye, O., and Tizabi, Y. (2014). Antidepressant effects of resveratrol in an animal model of depression. Behav. Brain Res. 268, 1-7. doi: 10.1016/j.bbr.2014.03.052

Isaacs, K. R., Anderson, B. J., Alcantara, A. A., Black, J. E., and Greenough, W. T. (1992). Exercise and the brain: angiogenesis in the adult rat cerebellum after vigorous physical activity and motor skill learning. J. Cereb. Blood Flow Metab. 12, 110-119. doi: 10.1038/jcbfm.1992.14

Kessler, R. C., Berglund, P., Demler, O., Jin, R., Koretz, D., Merikangas, K. R., et al. (2003). The epidemiology of major depressive disorder: results from the National comorbidity survey replication (NCS-R). JAMA 289, 3095-3105. doi: 10.1001/jama.289.23.3095

Kleim, J. A., Cooper, N. R., and VandenBerg, P. M. (2002). Exercise induces angiogenesis but does not alter movement representations within rat motor cortex. Brain Res. 934, 1-6. doi: 10.1016/s0006-8993(02) 02239-4

Lee, H., Ohno, M., Ohta, S., and Mikami, T. (2013). Regular moderate or intense exercise prevents depression-like behavior without change of hippocampal tryptophan content in chronically tryptophan-deficient and stressed mice. PLoS One 8:e66996. doi: 10.1371/journal.pone.0066996

Maes, M., Mihaylova, I., Kubera, M., and Ringel, K. (2012). Activation of cell-mediated immunity in depression: association with inflammation melancholia, clinical staging and the fatigue and somatic symptom cluster of depression. Prog. Neuropsychopharmacol. Biol. Psychiatry 36, 169-175. doi: 10.1016/j.pnpbp.2011.09.006

Malki, K., Lourdusamy, A., Binder, E., Payá-Cano, J., Sluyter, F., Craig, I., et al. (2012). Antidepressant-dependent mRNA changes in mouse associated with hippocampal neurogenesis in a mouse model of depression. Pharmacogenet. Genomics 22, 765-776. doi: 10.1097/FPC.0b013e328356fa90

Manosso, L. M., Moretti, M., Colla, A. R., Ribeiro, C. M., Dal-Cim, T. Tasca, C. I., et al. (2016). Involvement of glutamatergic neurotransmission in the antidepressant-like effect of zinc in the chronic unpredictable stress model of depression. J. Neural. Transm. 123, 339-352. doi: 10.1007/s00702-015 $-1504-3$

McCurdy, A. P., Boule, N. G., Sivak, A., and Davenport, M. H. (2017). Effects of exercise on mild-to-moderate depressive symptoms in the postpartum period: a meta-analysis. Obstet. Gynecol. 129, 1087-1097. doi: 10.1097/AOG. 0000000000002053

Menard, C., Hodes, G. E., and Russo, S. J. (2016). Pathogenesis of depression: insights from human and rodent studies. Neuroscience 321, 138-162. doi: 10.1016/j.neuroscience.2015.05.053

Niell, C. M., and Smith, S. J. (2004). Live optical imaging of nervous system development. Annu. Rev. Physiol. 66, 771-798. doi: 10.1146/annurev.physiol 66.082602 .095217

Nifosi, F., Toffanin, T., Follador, H., Zonta, F., Padovan, G., Pigato, G., et al. (2010). Reduced right posterior hippocampal volume in women with recurrent familial pure depressive disorder. Psychiatry Res. 184, 23-28. doi: 10.1016/j. pscychresns.2010.05.012

Otte, C., McCaffery, J., Ali, S., and Whooley, M. A. (2007). Association of a serotonin transporter polymorphism (5-HTTLPR) with depression, perceived stress, and norepinephrine in patients with coronary disease: the heart and soul study. Am. J. Psychiatry 164, 1379-1384. doi: 10.1176/appi.ajp.2007. 06101617

Ravaglia, G., Forti, P., Lucicesare, A., Pisacane, N., Rietti, E., Bianchin, M., et al. (2008). Physical activity and dementia risk in the elderly: findings from a prospective Italian study. Neurology 70, 1786-1794. doi: 10.1212/01.wnl. 0000296276.50595 .86 
Riddle, D. R., Sonntag, W. E., and Lichtenwalner, R. J. (2003). Microvascular plasticity in aging. Ageing Res. Rev. 2, 149-168. doi: 10.1016/s15681637(02)00064-8

Scheggi, S., Pelliccia, T., Ferrari, A., De Montis, M. G., and Gambarana, C. (2015). Impramine, fluoxetine and clozapine differently affected reactivity to positive and negative stimuli in a model of motivational anhedonia in rats. Neuroscience 291, 189-202. doi: 10.1016/j.neuroscience.2015.02.006

Shao, W. H., Li, C., Chen, L., Qiu, X., Zhang, W., Huang, C. X., et al. (2010). Stereological investigation of age-related changes of the capillaries in white matter. Anat. Rec. Hoboken 293, 1400-1407. doi: 10.1002/ar.21184

Swain, R. A., Harris, A. B., Wiener, E. C., Dutka, M. V., Morris, H. D., Theien, B. E., et al. (2003). Prolonged exercise induces angiogenesis and increases cerebral blood volume in primary motor cortex of the rat. Neuroscience 117, 1037-1046. doi: 10.1016/s0306-4522(02)00664-4

Tang, Y., Nyengaard, J. R., Andersen, J. B., Baandrup, U., and Gundersen, H. J. (2009). The application of stereological methods for estimating structural parameters in the human heart. Anat. Rec. Hoboken 292, 1630-1647. doi: 10.1002/ar.20952

Taragano, F. E., Allegri, R., Vicario, A., Bagnatti, P., and Lyketsos, C. G. (2001). A double blind, randomized clinical trial assessing the efficacy and safety of augmenting standard antidepressant therapy with nimodipine in the treatment of 'vascular depression'. Int. J. Geriatr. Psychiatry 16, 254-260. doi: 10.1002/ gps. 340

Weuve, J., Kang, J. H., Manson, J. E., Breteler, M. M., Ware, J. H., and Grodstein, F. (2004). Physical activity, including walking, and cognitive function in older women. JAMA 292, 1454-1461. doi: 10.1001/jama.292.12.1454
Yaghmaei, P., Parivar, K., Masoudi, A., Darab, M., and Amini, E. (2009). The effect of silybin on passive avoidance learning and pathological changes in hippocampal CA1 and DG regions in male Wistar rats offspring. J. Asian Nat. Prod. Res. 11, 514-522. doi: 10.1080/10286020902927864

Yang, S., Lu, W., Zhou, D. S., and Tang, Y. (2015). Enriched environment increases myelinated fiber volume and length in brain white matter of 18-month female rats. Neurosci. Lett. 593, 66-71. doi: 10.1016/j.neulet.2015.03.025

Yang, Y., Cao, J., Xiong, W., Zhang, J., Zhou, Q., Wei, H., et al. (2003). Both stress experience and age determine the impairment or enhancement effect of stress on spatial memory retrieval. J. Endocrinol. 178, 45-54. doi: 10.1677/joe.0. 1780045

Zheng, H., Liu, Y., Li, W., Yang, B., Chen, D., Wang, X., et al. (2006). Beneficial effects of exercise and its molecular mechanisms on depression in rats. Behav. Brain Res. 168, 47-55. doi: 10.1016/j.bbr.2005.10.007

Conflict of Interest Statement: The authors declare that the research was conducted in the absence of any commercial or financial relationships that could be construed as a potential conflict of interest.

Copyright (c) 2017 Chen, Zhou, Tan, Wang, Gao, Huang, Zhang, Jiang and Tang. This is an open-access article distributed under the terms of the Creative Commons Attribution License (CC BY). The use, distribution or reproduction in other forums is permitted, provided the original author(s) or licensor are credited and that the original publication in this journal is cited, in accordance with accepted academic practice. No use, distribution or reproduction is permitted which does not comply with these terms. 